All das begünstigt eine „mittige“ Rechtsprechung, die mehr integriert als polarisiert.

\section{Demokratievertrauen als Integrationsfaktor}

Das Integrationspotential unserer Verfassung ist kein Selbstläufer. Auch unter einer solchen Verfassung kann integrationspolitisch Vieles falsch gemacht werden - erst recht, wenn es um die Integration von Menschen geht, die nicht Bürger und also auch nicht Wähler sind und in Bezug auf die deshalb die Integrationsmechanismen der freiheitlichen Demokratie nur eingeschränkt zum Tragen kommen.

$\mathrm{Zu}$ den fehlerträchtigen Dispositionen gehört mangelndes Vertrauen in die Integrationskraft unserer freiheitlichen Demokratie. Daraus entsteht Angst, Reizbarkeit, leichte Provozierbarkeit und die Neigung zu symbolischen Gefechten anstelle geduldiger Arbeit an den realen Problemen, die sich stellen. All das ist Gift für die Integration unterschiedlicher Gruppen in einer Gesellschaft.

Warum dieses mangelnde Vertrauen? Die freiheitliche Demokratie als ein labiles Gebilde, der Erosion der Werte preis- gegeben, schwach in der Selbstbehauptung - diese Sichtweise hat starke Wurzeln in der europäischen und ganz besonders in der deutschen Kulturgeschichte. Sie hängt nicht nur mit der schon erwähnten Homogenitätslehre zusammen, sondern auch damit, dass die Demokratie, diese unmartialische Veranstaltung, als eine weibische, und damit eo ipso auch schwächliche, Angelegenheit verachtet war. Eine Assoziation, die gerade dieser Versammlung sicher besonders fernliegt, von der Weltbühne aber noch lange nicht abgetreten ist.

Die Deutschen haben ihre historische Demokratieverachtung in den Jahren seit dem Krieg überwunden. Das notwendige Vertrauen in die Stärke und Überzeugungskraft von Freiheit und Demokratie fehlt uns manchmal noch. Dieses Vertrauen ist keins, mit dem man sich zurücklehnen könnte. Die demokratische Verfassungsordnung ist keine Schlaraffenordnung, sondern die Ordnung, die es jedem erlaubt, an ihrer Verbesserung zu arbeiten, und die mehr als jede andere darauf ausgerichtet ist, aus Fehlern zu lernen. Ich wünsche Ihnen für Ihre Tagung die Arbeitsfreude und die Zuversicht, die man daraus beziehen kann.

\title{
Familiennachzug und Spracherwerb: Allgemeine Grundsätze und Rechtsprechung
}

\author{
Univ.-Prof. Dr. Margarete Schuler-Harms, Vorsitzende der Kom- \\ mission Recht der sozialen Sicherung, Familienlastenausgleich \\ im djb, Helmut-Schmidt-Universität, Hamburg
}

\section{Der Familiennachzug in Zahlen}

Im Aufenthaltsgrund des Familiennachzugs gehen Geschlecht und Familienzugehörigkeit eine besonders enge Verbindung ein. Einige Daten und Fakten sollen die große Bedeutung des Familiennachzugs im Rahmen der Migration und den hohen Anteil der Frauen im Familiennachzug verdeutlichen.

Der Familiennachzug bildete $2007^{1}$ den häufigsten Grund für die Zuwanderung nach Deutschland und in die Europäische Union. Nach Deutschland zogen rund 55.000 von rund 400.000 Ausländern aus familiären Gründen. Eine besondere Gruppe bilden dabei vor allem die sogenannten Drittstaatsangehörigen, also Ausländer ohne Staatsangehörigkeit in einem EU-Mitgliedstaat. Rund 29 Prozent der einreisenden Drittstaatsangehörigen zogen 2007 zu Ehepartnern oder anderen Familienangehörigen nach. Den Hauptanteil bildeten dabei Türkinnen und Türken, von denen nahezu die Hälfte zum Zweck des Familiennachzugs einreiste. ${ }^{2}$ Kein anderer Aufenthaltsgrund wird von Drittstaatsangehörigen annähernd häufig geltend gemacht. Knapp 20 Prozent der Drittstaatsangehörigen kommen, um eine Schulbildung, eine Ausbildung, ein Studium oder einen Sprachkurs zu absolvieren, ca. 15,3 Prozent bezwecken die Aufnahme einer Beschäftigung und etwa 2,6 Prozent reisen aus humanitären Gründen ein. ${ }^{3}$ Die Zuwanderung aus familiären Gründen unterscheidet sich von anderen Zuwanderungsgründen auch dadurch, dass der Aufenthalt in der Bundesrepublik regelmäßig langfristig oder gar auf Dauer angelegt ist.

Der Anteil der Frauen im Familiennachzug übersteigt den der Männer deutlich. ${ }^{4}$ Der Gesamtanteil der Frauen an den Zuzügen ins Bundesgebiet liegt seit etwa 15 Jahren konstant bei 40 bis 43 Prozent, der Anteil der Migrantinnen beim Zuzug zu Beschäftigungszwecken bei etwa einem Drittel. Demgegenüber wurden von den insgesamt ca. 55.000 aus familiären Gründen erteilten Aufenthaltstiteln über die Hälfte (knapp 52 Prozent, im Vorjahr 55 Prozent) an nachziehende Ehefrauen erteilt. Ein knappes Viertel (22,3 Prozent) ging an nachziehende Ehemänner und etwa ebenso viel (22,4 Prozent) an nachziehende Kinder. Der Anteil sonstiger Familienangehöriger lag bei 3,6 Prozent und umfasste ganz überwiegend den

1 Vgl. Bundesamt für Ausländer und Flüchtlinge (BAMF), Migrationsbericht 2007, $30 \mathrm{ff} .$, abrufbar unter www.bmi.bund.de.

2 Vgl. BAMF, Migrationsbericht 2007, 120 (Übersichten). Der Anteil der Türkinnen und Türken am Ehegatten- und Familiennachzug insgesamt betrug 33,4\% im Jahr 1997 und immerhin noch 21,9\% im Jahr 2007, vgl. BAMF, Migrationsbericht 2007, 121.

3 BAMF, Migrationsbericht 2007, Übersicht 32.

4 BAMF, Migrationsbericht 2007, $30 \mathrm{f}$. 
sorgeberechtigten Elternteil eines deutschen minderjährigen Kindes. ${ }^{5}$ Vor allem der Aufenthaltsgrund des Ehegattennachzugs ist also für Frauen von spezifischer Bedeutung.

\section{Rechtliche Grundlagen}

Das Gemeinschaftsrecht regelt den Familiennachzug in zwei Richtlinien. Die RL 2004/38/EG (FreizügigkeitsRL/EU) regelt den Nachzug von Staatsangehörigen aus Drittstaaten zu EUStaatsangehörigen (Unionsbürgern), die RL 2003/86/EG (FamilienzusammenführungsRL) den Nachzug von Drittstaatern zu Angehörigen, die ihrerseits aus einem Drittstaat stammen. ${ }^{6}$ Den Zuzug von Ausländern zu Staatsangehörigen des Einreisestaates erfassen die Richtlinien als rein innerstaatliche Sachverhalte grundsätzlich nicht.

Deutschland hat diese Richtlinien in zwei Schritten umgesetzt. Mit dem Zuwanderungsgesetz trat zum 1. Januar 2005 das Aufenthaltsgesetz (AufenthG) in Kraft und regelte in $\mathbb{S} \mathbb{S}$ 27-36 den Zuwanderungsgrund des Familiennachzugs. Im Richtlinienumsetzungsgesetz (RLUmsG), in Kraft getreten am 28. Januar 2007, modifizierte der Gesetzgeber die noch jungen Regeln zum Familiennachzug und verschärfte dabei die Bedingungen für den Ehegattennachzug ganz erheblich.

Der in Deutschland lebende Ehepartner oder Familienangehörige muss Deutscher oder im Besitz eines für den Familiennachzug geeigneten Aufenthaltstitels sein. Außerdem muss ausreichend Wohnraum verfügbar und der Lebensunterhalt auch für den nachziehenden Ehepartner gesichert sein. Diese Anforderungen gelten für den Familiennachzug allgemein und blieben durch das RLUmsG unberührt. Für den Ehegattennachzug, der im Folgenden näher betrachtet werden soll, gelten zusätzliche Anforderungen.

Wer als Ehegatte zu einem in Deutschland lebenden Drittstaater, Unionsbürger oder Deutschen nachziehen will, muss nach geltendem Recht eine „Ehe“ im Sinne des Aufenthaltsrechts führen. Dabei hat das deutsche Ausländerrecht, wonach bei Mehrehen nur eine Ehefrau nachzugsberechtigt ist, europarechtliche Verstärkung erfahren: Die FamiliennachzugsRL rechtfertigt ausdrücklich eine restriktive Handhabung des Familiennachzugs bei Mehrehen aus „Achtung der von den Mitgliedstaaten anerkannten Werte und Grundsätze, insbesondere der Rechte von Frauen und Kindern “. ${ }^{7}$ Auch sogenannte Imam-Ehen, die nicht standesamtlich, sondern nach islamischem Kodex religiös geschlossen wurden, berechtigen nicht zum Familiennachzug. ${ }^{8}$ Dagegen stellt das AufenthG die gleichgeschlechtliche Lebenspartnerschaft nach dem Lebenspartnerschaftsgesetz der Ehe gleich. ${ }^{9}$ Der 2007 eingeführte $\mathbb{S} 27$ Abs. 1a AufenthG schließt erstmals explizit den Nachzug für Schein- bzw. Zweckehen und Zwangsehen aus. ${ }^{10}$

Das RLUmsG von 2007 bindet den Ehegattennachzug an weitere Bedingungen. Beide Ehegatten müssen ein Mindestalter von 18 Jahren erreicht haben und der nachzugswillige Ehegatte sich zumindest auf einfache Art in deutscher Sprache verständigen können ( $\mathbb{S} 30$ Abs. 1 Satz 1 Nr. 1, 2 AufenthG). Allerdings gelten diese Bedingungen nur für bestimmte Fallkonstellationen (vgl. $\mathbb{S} 30$ Abs. 1 Sätze 2, 3 AufenthG). Sie

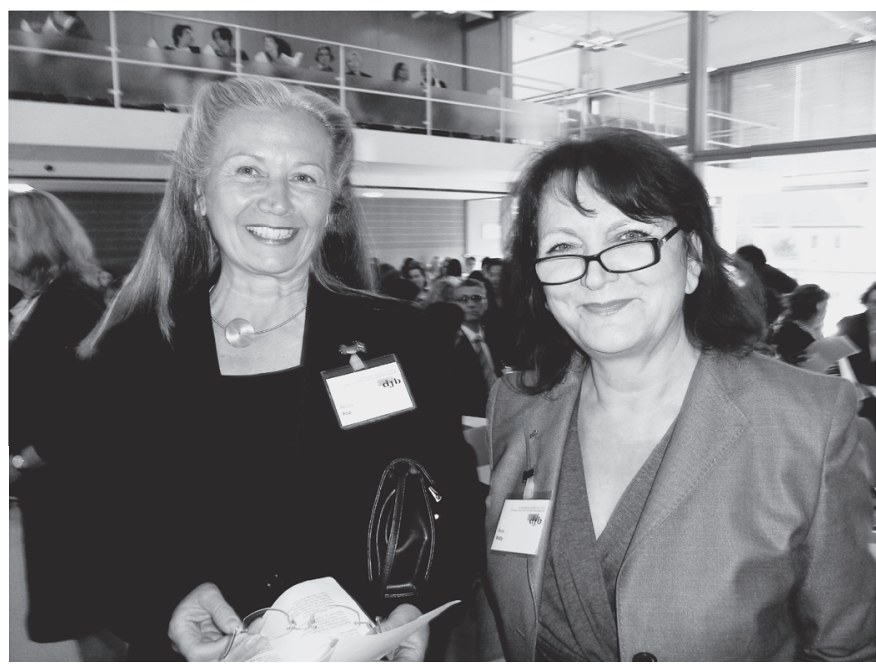

$\Delta$ Vors. Richterin am OLG Ramona Pisal (links) mit Rechtsanwältin Renate Maltry (djb-Vizepräsidentinnen) (Foto: AG).

entfallen, wenn der in Deutschland lebende Ehepartner über einen Aufenthaltstitel als Hochqualifizierter verfügt oder sich zum Zwecke der Forschung oder der Aufnahme einer selbständigen Tätigkeit in Deutschland aufhält und die Ehe bereits vor seiner Einreise bestand. Beruht die Aufenthaltserlaubnis auf einer langfristigen Aufenthaltsberechtigung in einem anderen Mitgliedstaat der EU und bestand dort auch schon die eheliche Lebensgemeinschaft, so entfallen beim Aufenthalt in Deutschland ebenfalls die besonderen Nachzugsbedingungen des $\mathbb{3} 30$ Abs. 1 Nr. 1, 2 AufenthG. Auf den Nachweis von Sprachkenntnissen wird verzichtet für den Nachzug zu Ausländern, die nach ihrer Staatsangehörigkeit visumfrei nach Deutschland einreisen dürfen. Dieses Recht haben Staatsangehörige aus den EU- und den EWR-Staaten ( $\mathbb{1} 12$ FreizügG/EU) sowie zum Beispiel aus der Schweiz, Australien, Israel, Japan, Kanada, Südkorea, Neuseeland und den Vereinigten Staaten. Neben solchen, teilweise wirtschaftspolitisch motivierten Ausnahmen ${ }^{11}$ ist auch der Nachzug zu Ausländern mit humanitärem Aufenthaltsgrund privilegiert. Eines Nachweises bedarf es außerdem dann nicht, wenn der nachziehende Ehepartner wegen Krankheit oder Behinderung nicht in der Lage ist, einfache Kenntnisse der deutschen Sprache nachzuweisen, oder wenn „erkennbar geringer Integrationsbedarf“ besteht, etwa weil der Ehepartner über einen Hochschul- oder Fachhochschulabschluss oder eine entsprechende Qualifikation verfügt oder eine Erwerbstätigkeit ausübt, die regelmäßig eine solche

5 BAMF, Migrationsbericht 2007, 120 (Übersichten) und 126.

6 Vgl. zur letzteren EuGH Urt. v. 25.7.2008 - C-127/08 - Metock.

7 Erwägungsgrund 11 und Art. 4 Abs. 4 der RL 2003/86/EG.

8 Eberle, in:Storr/Wenger/Eberle/Albrecht/Harms, Kommentar zum Zuwanderungsrecht, § 27 AufenthG Rn. 10; BVerwG Urt. v. 22.2.2005 - 1 C 17/03, InfAusIR 2005, 397.

$9 \$ 27$ Abs. 2 AufenthG.

10 Amtl. Begr. zum RLUmsG, BT-Drucks. 16/5065, 3 und 170. Zur Begrifflichkeit vgl. Veronika Arendt-Rojahn, Die Zweckehe im Ausländerrecht, FF 2009, 14 ff., die die „Scheinehe“ als Unterfall der „Zweckehe" behandelt.

11 Amtl. Begr., BT-Drucks. 16/5065, 174 f. 
Qualifikation voraussetzt, und wenn im Einzelfall die Annahme gerechtfertigt ist, dass der Ehegatte sich ohne staatliche Hilfe in das wirtschaftliche, gesellschaftliche und kulturelle Leben in Deutschland integrieren wird. ${ }^{12}$

Die Verschärfungen des Ehegattennachzugs im RLUmsG von 2007 und ihre Beschränkung auf bestimmte aufenthaltsrechtliche Konstellationen waren schon im Gesetzgebungsverfahren heftig umstritten. Wegen der Brisanz der Regelungen und deren besonderer genderspezifischer Bedeutung konzentrieren sich die folgenden Ausführungen auf diese neuen Anforderungen. Die aktuelle Bestandsaufnahme unter Einbezug der einschlägigen Rechtsprechung ergibt hierzu ein gemischtes Bild.

\section{Neue Anforderungen für den Ehegattennachzug}

\section{Bestehen einer Ehe}

\section{Keine Schein- bzw. Zweckehe}

Fehlt es am Willen der Beteiligten zur Herstellung einer ehelichen Lebensgemeinschaft, liegt eine Scheinehe, nach anderer Diktion eine Zweckehe vor und ist ein Ehegattennachzug ausgeschlossen ( $\$ 27$ Abs. 1a Nr. 1 AufenthG). Schon im Gesetzgebungsverfahren zum Erlass des RLUmsG wurde die Sorge geäußert, dass binationale Ehen und Ehen zwischen Ausländern künftig einem Generalverdacht und intensiveren Nachforschungen der Ausländerbehörden ausgesetzt sein könnten. ${ }^{13}$ Die Rechtsprechung hält jedoch an dem Grundsatz fest, dass die Ausländerbehörden und Auslandsvertretungen auch weiterhin nur bei begründetem Verdacht oder - in anderer Diktion - bei atypischen Abweichungen vom Regelfall zu solchen Nachforschungen berechtigt seien. In diesem Falle haben die Beteiligten weiterhin die Obliegenheit zur Mitwirkung an der Aufklärung (vgl. $\$ 82$ AufenthG). ${ }^{14}$ Diese Grundsätze formuliert auch Art. 16 Abs. 4 FamilienzusammenführungsRL. ${ }^{15}$

Sehr umstritten ist derzeit jedoch die Frage, wer die materielle Beweislast für den Fall trägt, dass sich der Herstellungswille der Beteiligten oder dessen Fehlen nicht nachweisen lässt. Nach Auffassung eines Teils der Literatur lassen Wortlaut und Systematik der Regelung wie auch ihre Bezüge zur FamilienzusammenführungsRL darauf schließen, dass der Gesetzgeber die Beweislast gegenüber dem älteren AusländerR ändern wollte. Hiernach hätten die Behörden den Nachzug zu gestatten, wenn der Nachweis für einen fehlenden Herstellungswillen nicht gelänge. ${ }^{16}$ Die Rechtsprechung und ein anderer Teil der Literatur sind anderer Ansicht. ${ }^{17}$ Einige Verwaltungsgerichte und die ersten Oberverwaltungsgerichte gehen davon aus, dass die materielle Beweislast auch nach neuem Recht bei der nachzugswilligen Ausländerin respektive dem nachzugswilligen Ausländer liegt, es ihnen also gelingen muss, etwaige Zweifel am Herstellungswillen zu zerstreuen.

\section{Keine Anhaltspunkte für eine Zwangsehe}

Ein Ehegattennachzug ist gem. $\$ 27$ Abs. 1a Nr. 2 AufenthG ausgeschlossen, wenn tatsächliche Anhaltspunkte die Annahme begründen, dass einer der Ehegatten zur Eingehung einer
Ehe genötigt wurde. Der Ausschlussgrund bildet Teil eines Gesamtpakets von Maßnahmen zur Bekämpfung von Zwangsverheiratungen, zu denen auch die in $\mathbb{3} 30$ Abs. 1 AufenthG normierten Nachzugsbedingungen (s.u. „Mindestnachzugsalter“ und „Nachweis sprachlicher Fertigkeiten“) zählen.

Die Vorschrift wirkt Zwangsverheiratungen allerdings nur sehr begrenzt entgegen und ist auch aus anderen Gründen problematisch. Sie erfasst erstens nicht die Fälle der innerhalb Deutschlands oder zwischen Deutschen geschlossenen Zwangsehen und schützt auch nicht gegen die sogenannte Urlaubsheirat, bei der das Opfer zu Heiratszwecken ins Ausland verbracht oder in den Ferien dort verheiratet wird. Der Ausschlussgrund erweist sich zweitens als wirkungslos, wenn aus der Zwangsehe ein Kind hervorgeht, das die deutsche Staatsangehörigkeit oder ein Familiennachzugsrecht sowohl selbst besitzt als auch für die Mutter begründet. Drittens reichen nach dem Gesetz für den Nachzugsausschluss „tatsächliche Anhaltspunkte“ aus, die die Annahme begründen, dass einer der Ehegatten zur Eingehung der Ehe genötigt wurde“ ( $\mathbb{S} 27$ Abs. 1a Nr. 2 AufenthG). Die ohnehin schwierige Abgrenzung von Zwangs- und arrangierter Ehe bleibt danach unsicher. ${ }^{18}$ Rechtsprechung ist zu diesem Ausschlussgrund bislang nicht zu verzeichnen.

\section{Mindestnachzugsalter}

Die schon angesprochenen Nachzugsbedingungen des $\mathbb{} 30$ Abs. 1 AufenthG sollen zum einen dem Zweck der besseren Integration von sogenannten Drittstaatsangehörigen dienen, zum anderen sind sie wiederum durch den Zweck der präventiven Verhinderung von Zwangsehen motiviert. ${ }^{19}$

12 BT-Drucks. 16/5065, 175 unter Bezug auf $\$ 4$ Integrationsverordnung.

13 Vgl. z.B. Fischer-Lescano, Kritische Justiz 2006, 236 (239 f.); Eberle, in: Storr u.a., $\$ 27$ Rn. 16 für binationale Ehen.

14 Vgl. z.B. HessVGH, U.v. 3.9.2008-11 B 169o/o8, juris; OVG BerlinBrandenburg, U. v. 29.1.2009- 2 B 11.08, juris. Ausführlich Müller, in: Hofmann/Hoffmann (Hrsg.), Handkommentar Ausländerrecht, 2008, § 27 AufenthG Rn. $27 \mathrm{f}$.

15 So auch Bericht der Kommission an das Europäische Parlament und an den Rat über die Anwendung der Richtlinie 2003/86/EG des Rats betreffend das Recht auf Familienzusammenführung vom 8.10.2008, Bericht 2008 NaN 52008DC0610, Gliederungspunkt 4.4.1.

16 Vgl. Müller, in: Hofmann/Hoffmann, Handkommentar Ausländerrecht, $§ 27$ AufenthG Rn. 28; Huber/Göbel-Zimmermann, Ausländerund Asylrecht, 2. Auflage 2008, Rn. 712 mit Bezug auf Oertmann, InfAusIR 2008, 17 (21).

17 OVG Berlin-Brandenburg, Urt. v. 29.1.2009-2 B 11.08, juris, m.w.Nw. zum Streitstand (Rn. 31 ff.); HessVGH Urt. v. 3.9.2008-11 B 169o/o8, juris; VG Berlin, Urt. v. 19.12.2007 - VG 5 V 22.07 - juris; Katharina Breitkreutz/Boris Franßen-de la Cerda/Christoph Hübner, Das Richtlinienumsetzungsgesetz und die Fortentwicklung des deutschen Aufenthaltsrechts, Teil 2, ZAR 2007, 381 (382) unter Bezug auf die Signalfunktion der Vorschrift, mit der „einerseits das Unrechtsbewusstsein der Betroffenen und andererseits das Problembewusstsein der Rechtsanwender erhöht werden" solle.

18 Schuler-Harms, Zwangsverheiratung und Aufenthaltsrecht, in: Klaus Barwig/Stephan Beichel-Benedetti/Gisbert Brinckmann (Hrsg.), Perspektivwechsel im Ausländerrecht?, 2007, 276 f.; sich anschließend Müller, in: Hofmann/Hoffmann, Handkommentar Ausländerrecht, § 27 AufenthG Rn. 24.

19 BT-Drucks. 16/5065, $172 \mathrm{f}$. 


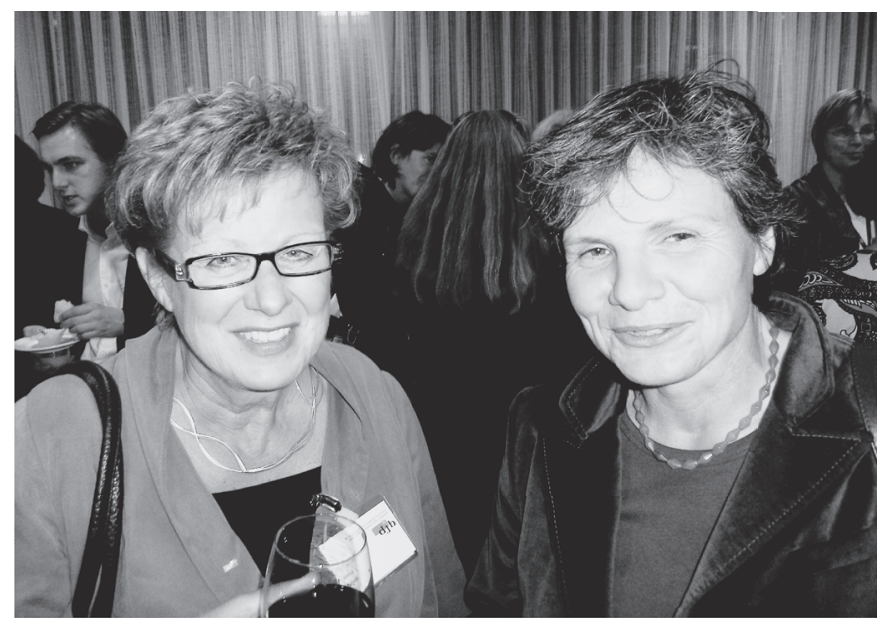

$\Delta$ Rechtsanwältin und frühere Geschäftsführerin des djb Martina Bosch (links) mit Prof. Dr. Dagmar Oberlies (Foto: AG).

Das Mindestnachzugsalter von 18 Jahren wurde bei seiner Einführung heftig kritisiert, ist aber europarechtskonform. Die Familienzusammenführungsrichtlinie erlaubt die Festlegung eines Mindestnachzugsalters von höchstens 21 Jahren zur Förderung der Integration und zur Vermeidung von Zwangsehen (Art. 4 Abs. 4 RL 2003/86/EG). Eine Bestandsaufnahme der Europäischen Kommission hat ergeben, dass die meisten Mitgliedstaaten ein Mindestnachzugsalter festlegen, fünf davon sogar mit der Obergrenze von 21 Jahren, und dies mit dem Zweck der Vermeidung von Zwangsehen begründen. ${ }^{20}$ Auch verfassungsrechtlich ist das vorübergehende Einreisehindernis des Mindestnachzugsalters von 18 Jahren nicht zu beanstanden. ${ }^{21}$ Die Regelung ist hinreichend klar und das Hindernis vorübergehend, denn bei einem dem deutschen Familienrecht entsprechenden Mindestheiratsalter von 16 Jahren (vgl. \ 1303 Abs. 2 BGB) ergibt sich eine maximale Wartezeit von zwei Jahren. Das Gesetz enthält überdies in \30 Abs. 2 Satz 1 AufenthG eine Härteklausel. Die oben beschriebenen Ausnahmen für die erwähnten Aufenthaltstitel mögen politisch fragwürdig sein, ${ }^{22}$ doch dürften sie noch im Gestaltungsspielraum des Gesetzgebers liegen und keine verfassungswidrige Diskriminierung begründen. Interessanterweise sprechen sich gerade Wissenschaftlerinnen und Wissenschaftler mit Migrationshintergrund eher für als gegen das Mindestnachzugsalter aus. ${ }^{23}$ Aktuelle Rechtsprechung zu dieser Nachzugsbedingung ist nicht zu verzeichnen.

\section{Nachweis sprachlicher Fertigkeiten}

Sehr viel problematischer ist von Beginn an das Erfordernis der Verständigungsmöglichkeit auf einfache Art in deutscher Sprache ( $\mathbb{S} 30$ Abs. 1 Nr. 2 AufenthG) gesehen worden. Die FamilienzusammenführungsRL sieht entsprechende mitgliedstaatliche Regelungen nicht einmal fakultativ vor. Gestützt wird die deutsche Regelung auf Art. 7 Abs. 3 RL 2003/86/EG, der es den Mitgliedstaaten erlaubt, Drittstaatsangehörigen Integrationsmaßnahmen aufzuerlegen. Das Erfordernis sprachlicher Fertigkeiten im deutschen Zuwanderungsrecht ist freilich nicht als Integrationsmaßnahme konzipiert, der der nachziehende Ehegatte auch nach seiner Einreise nachkommen könnte. Vielmehr wird in der europarechtlichen Diktion eine Integrationsbedingung formuliert. Ist diese Bedingung nicht erfüllt, weil die sprachlichen Fertigkeiten nicht zur Überzeugung der Auslandsvertretungen in den Herkunftsländern nachgewiesen werden, so sind die Erteilung eines Visums zum Ehegattennachzug und die Einreise ins Bundesgebiet ausgeschlossen.

Nach den Erhebungen der Europäischen Kommission sind nur in zwei weiteren Mitgliedstaaten Integrationsmaßnahmen zugleich als Integrationsbedingungen ausgestaltet worden. Das niederländische Recht, dem das deutsche nachgebildet wurde, verlangt von Familienangehörigen die erfolgreiche Teilnahme an einem nur im Herkunftsland zu absolvierenden ersten Integrationstest, der sich auf die Kenntnis der niederländischen Sprache und Gesellschaft erstreckt. In Frankreich müssen Nachzugswillige einen „Aufnahme- und Integrationsvertrag “ unterschreiben, aufgrund dessen sie zur Teilnahme an Kursen in Staatsbürgerkunde und erforderlichenfalls an Sprachkursen verpflichtet werden. ${ }^{24}$

\section{Vereinbarkeit mit Gemeinschaftsrecht}

Die Europarechtskonformität der in $\mathbb{S} 30$ Abs. 1 Nr. 2 AufenthG geregelten Zuzugsbedingung war und ist heftig umstritten. ${ }^{25}$ Die Europäische Kommission verneint diese in ihrem Bericht an den Rat vom Juli 2008 nicht gänzlich, formuliert aber besondere Anforderungen. Die Zulässigkeit der Maßnahmen hänge davon $a b$, inwieweit der Zugang zu solchen Kursen und Tests gewährleistet sei, wie sie konzipiert und/oder organisiert seien (Kursunterlagen, Gebühren, Veranstaltungsort) und ob mit solchen Maßnahmen und ihren Auswirkungen auch andere Zwecke als die Integration verfolgt, etwa durch hohe Gebühren Familien mit geringem Einkommen vom Nachzug ausgeschlossen würden. Auch Verfahrensrechte zur Ermöglichung der Rechtsdurchsetzung fordert die Kommission ein. Diese Ausführungen verdeutlichen eine gehörige Skepsis. Sie zeigt sich erneut in den Schlussfolgerungen des Berichts, in denen die Kommission den zu wenig verbindlichen Charakter der Richtlinie und den großen Ermessensspielraum der Mitgliedstaaten beklagt und in diesem Zusammenhang

20 Vgl. Bericht der Kommission an das EP und an den Rat über die Anwendung der RL 2003/86/EG des Rates betreffend das Recht auf Familienzusammenführung vom 8.10.2008, Dokument 52008DC0610, unter 4.1.

21 Strenger z.B. Thorsten Kingreen, ZAR 2007, S. 13 (14 ff.), allerdings für ein Mindestnachzugsalter von 21 Jahren; Ralph Göbel-Zimmermann, ZAR 2008, 169 (170 f.); Fischer-Lescano, Kritische Justiz 2006, 236 (240 f.).

22 Dies gilt insbesondere für die Privilegierung von Ausländern aus visafreien Staaten gegenüber Deutschen, vgl. oben „Mindestnachzugsalter" am Ende.

23 Vgl. Ahmed Toprak, Das schwache Geschlecht - Die türkischen Männer, 2005, 176 f.; Necla Kelek, Die fremde Braut, 2005, 233.

24 Kommission, Bericht über die Anwendung der RL 2003/86/EG, unter 4.3.4.

25 Kritik z.B. bei Hoffmann, in: Hofmann/Hoffmann, Ausländerrecht Handkommentar, § 30 AufenthG Rn. 29; großzügiger Kay Hailbronner, FamRZ 2008, 1583 (1587 f.). 


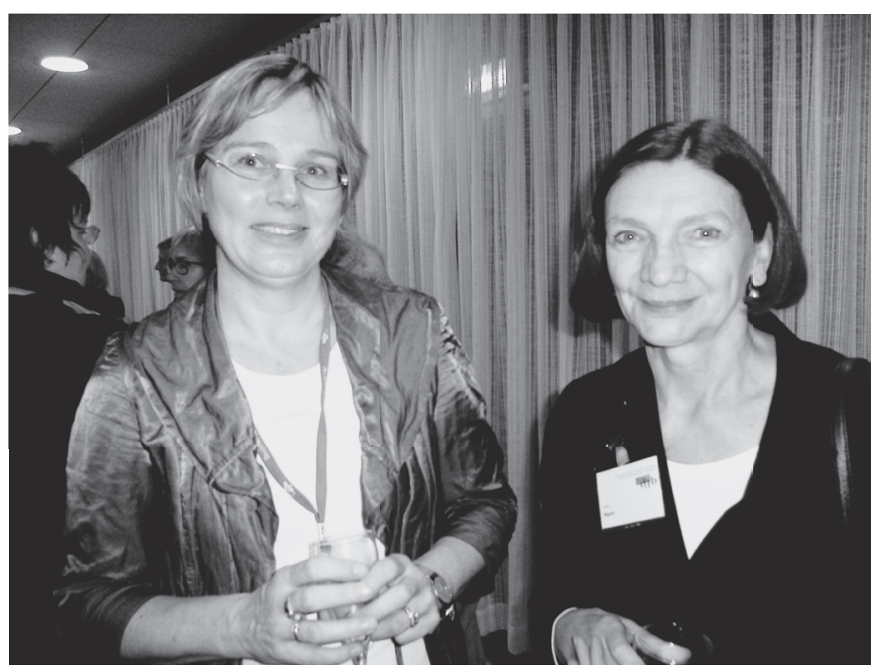

$\triangle$ Abteilungsleiterin im BMFSFJ Eva Maria Welskop-Deffaa (links) mit djbPräsidentin Rechtsanwältin und Notarin Jutta Wagner (Foto: AG)

sowohl die Integrationsmaßnahmen als auch das Mindestnachzugsalter besonders erwähnt. ${ }^{26}$

\section{Anforderungen an sprachliche Fertigkeiten und deren Nachweis}

Ein Blick auf die aktuelle Rechtsprechung zeigt, dass offenbar eine - nicht immer reflektierte - Unsicherheit über den Standard der zu fordernden Sprachkenntnisse und Verständigungsmöglichkeiten besteht. Gefordert sind ausreichende Grundkenntnisse der deutschen Sprache auf der Kompetenzstufe 1 des Gemeinsamen Europäischen Referenzrahmens des Europarats - GER. ${ }^{27}$ Die Stufe A1 GER umfasst als unterstes Sprachstandsniveau die folgenden sprachlichen Fertigkeiten:

„Kann sich mit einfachen, überwiegend isolierten Wendungen über Menschen und Orte äußern. Kann sich auf einfache Art verständigen, doch ist die Kommunikation völlig davon abhängig, dass etwas langsamer wiederholt, umformuliert und korrigiert wird. Kann einfache Fragen stellen und beantworten, einfache Feststellungen treffen und auf solche reagieren, sofern es sich um unmittelbare Bedürfnisse oder um sehr vertraute Themen handelt, z.B. wo sie/er wohnt, welche Leute sie/er kennt, oder welche Dinge sie/er hat. “28

Nach anderer Darlegung umfasst die Prüfung

„insgesamt die Sprachkenntnis von ca. 650 Wörtern, von denen lediglich etwa 300 Wörter aktiv beherrscht werden sollten.“29

Was aber wird im Einzelnen verlangt? Nach einer Entscheidung des VG Berlin setzt eine Verständigung auf einfache Art in deutscher Sprache voraus, dass der Ausländer Sätze mit Subjekt, Prädikat und Objekt bilden und entsprechende Sätze mit geläufigen Alltagsbegriffen mehr als nur selten verstehen kann. ${ }^{30}$ Das OVG Berlin-Brandenburg verneint diese grammatikalischen Anforderungen, verlangt aber die Fähigkeit zur einfachen schriftlichen Verständigung in deutscher Sprache. ${ }^{31}$ Diese Anforderung begründet das Gericht unter anderem mit dem Zweck der Integrationsförderung, für den die Fähigkeit, einfache Texte in deutscher Sprache lesen und schreiben zu können, von besonderer Bedeutung sei. Nicht nur im Umgang mit Behörden, sondern auch zur Teilhabe am sonstigen sozialen Miteinander und am wirtschaftlichen Leben in Deutschland erscheine die Fähigkeit zu lesen und zu schreiben geradezu unabdingbar. Wer also die mündliche Verständigung in deutscher Sprache erlernt hat und nach Deutschland zum (möglicherweise deutschen) Ehepartner reisen möchte mit dem Vorsatz, dort die Kenntnisse in deutscher Sprache zu vertiefen und in deutscher Schrift zu erlernen, wird am Zweck der Integrationsförderung scheitern. Vom Zweck der Bekämpfung von Zwangsheiraten ist in diesen Entscheidungen nicht mehr die Rede.

Noch weniger ist geklärt, welchen Anforderungen der Nachweis dieser Sprachkenntnisse genügen muss. Die sprachlichen Fertigkeiten sollen nach einer Ansicht durch den Besuch eines entsprechenden Sprachkurses der Goethe-Institute oder von diesen beauftragter oder zertifizierter Sprachschulen und das Zertifikat A1 „Deutsch Start 1“ nachgewiesen werden. ${ }^{32}$ Da ein solcher Nachweis gesetzlich nicht gefordert ist, kommt es nach richtiger Auffassung auf die tatsächlichen Sprachkenntnisse an. Diese soll nach den Ausführungen des OVG Nordrhein-Westfalen die Ausländerbehörde auch dann nachprüfen können, wenn ein entsprechendes Zertifikat vorliegt und die Auslandsvertretung vor Visumserteilung die sprachlichen Fertigkeiten bereits bejaht hat. ${ }^{33}$ Nach Ansicht des OVG Berlin-Brandenburg, das sich in der angesprochenen Entscheidung am ausführlichsten mit der Frage beschäftigt, soll der Nachweis nicht auf die Vorlage eines entsprechenden Zertifikats beschränkt sein, sondern sollen offensichtlich vorhandene, im Gespräch mit den behördlichen Mitarbeitern belegte Sprachkenntnisse genügen. Liegt ein entsprechendes Zertifikat vor, genügt dieses. ${ }^{34}$

26 Kommission, Bericht über die Anwendung der RL 2003/86/EG, unter 5. (Schlussfolgerungen).

27 Hinweise des BMI zum Richtlinienumsetzungsgesetz, Stand 18.12.2007, veröffentlicht unter www.bmi.bund.de, Rz. 210 ff.; BAMF, Migrationsbericht 2007, 117; Auslandsvertretung im Fall des OVG Lüneburg, B.v. 27.7.2009 - 11 ME 171/o9, Rn. 13, 17, juris; OVG BerlinBrandenburg, Urt. v. 28.4.2009-2 B 6/08, juris.

28 Zitiert nach OVG Brandenburg, 28.4.2009, - 2 B 6/o8, juris, Rn. 25; vgl. a. Bundesministerium des Innern, Migration und Integration, 2008, 90, abrufbar unter www.bmi.bund.de; Göbel-Zimmermann, ZAR 2008, 169 (171).

29 BAMF, Migrationsbericht 2007, 117; BT-Drucks. 16/9137, 5.

30 VG Berlin v. 19.12.2007- 5 V 22.07, juris.

31 OVG Berlin-Brandenburg 28.4.2009- 2 B 6/o8, Rn. 27, juris; vgl. demgegenüber Göbel-Zimmermann, ZAR 2008, 169 (171): Er (der Ausländer, MSH) ,sollte“ zudem auch ein wenig Deutsch schreiben können, d.h. z.B. in der Lage sein, auf Formularen in Hotels Name, Adresse, Nationalität usw. eintragen können (Anführungszeichen im Original unter Bezug auf den Gemeinsamen Referenzrahmen).

32 BMAF, Migrationsbericht 2007, 117 („grundsätzlich“) in Ländern, in denen Sprachprüfungen „Start Deutsch 1“ angeboten werden. Mit dieser Tendenz offenbar auch VGH Baden-Württemberg, B. v. 10.3.2009 - 11 S 2990/o8, juris, unter B.I.1.a.

33 OVG Nordrhein-Westfalen, B. v. 3.6.2009-18 B 1828/o8, juris, Rn. 10, auch zur Befugnis, einen weiteren Sprachtest zu verlangen.

34 OVG Berlin-Brandenburg, 24.4.2009, Rn. 28; ausführlich Göbel-Zimmermann, ZAR 2008, 169 (172). 
Auch wenn diese Entscheidung vernünftige Anforderungen formuliert, ist die Rechtsunsicherheit über das verlangte Sprachniveau und seinen Nachweis doch noch zu groß. Der Verwaltungsvollzug in den Auslandsvertretungen der jeweiligen Herkunftsländer verlangt nach klaren Maßstäben für Standard und Nachweis der Sprachkenntnisse. Unklare Maßstäbe und unsichere Rechtsprechung führen zu Disparitäten in der Kontrolle der Zuzugsbedingung. Die Folgen für den Ehegattennachzug sind unter Umständen nicht nur vorübergehend, sondern existentiell. Die Hürden für den Ehegattennachzug werden unnötig erhöht.

\section{Möglichkeiten zum Erwerb der sprachlichen Fertigkeiten in Deutschland}

Ein dritter Problemkreis betrifft die Möglichkeiten, die erforderlichen sprachlichen Fertigkeiten nicht im Ausland, sondern unmittelbar in Deutschland zu erwerben. Eine noch nicht ganz ausgelotete Möglichkeit hierzu ist die Schwangerschaft der nachzugswilligen Ehefrau. Eine andere Möglichkeit zum Spracherwerb für den erstrebten Daueraufenthalt könnte ein Besuchsvisum bieten.

Im Rahmen der Prüfung von Anträgen auf ein sogenanntes Schengen-Visum ${ }^{35}$ prüfen die Auslandsvertretungen auch, ob der Aufenthalt eine Beeinträchtigung oder Gefährdung berechtigter Interessen der Bundesrepublik oder anderer Mitgliedstaaten mit sich bringt. Berechtigte Interessen sind insbesondere dann berührt, wenn der Ausländer eine Ausreise zum Ablauf des Besuchsvisums gar nicht beabsichtigt, das Besuchsvisum also zum Zwecke illegaler Einwanderung missbrauchen will. Anna Lübbe hat jüngst beschrieben, dass die Verwaltungsgerichte die Absicht zur illegalen Einwanderung regelmäßig bei Eheleuten bejahen, die ein Besuchsvisum beantragen und die darlegen, in Deutschland Sprachkurse besuchen zu wollen. Eine Verfassungsbeschwerde gegen diese Rechtsprechung sei beim BVerfG anhängig. ${ }^{36}$

Für die große Gruppe nachziehender türkischer Ehepartner ${ }^{37}$ könnte die Entscheidung des EuGH in Sachen Soysal vom 19. Februar $2009^{38}$ neue Möglichkeiten eröffnen. Herr Soysal und Herr Savatli, zwei für ein internationales Gütertransportunternehmen tätige Fernfahrer, wandten sich gegen die Visumspflicht zur vorübergehenden Einreise in das Bundesgebiet. Der EuGH bestätigt auf Vorlage des OVG BerlinBrandenburg, ${ }^{39}$ dass die seit 1980 bestehende Visumspflicht den freien Dienstleistungsverkehr unzulässig beschränke. Die einschlägigen, für die Beziehungen der EWG und der Türkei bestehenden Vorschriften verböten, ein Visum für die Einreise türkischer Staatsangehöriger in das Hoheitsgebiet eines Mitgliedstaats zu verlangen, um dort Dienstleistungen für ein in der Türkei ansässiges Unternehmen erbringen zu wollen, wenn eine solche Visumspflicht zur Zeit des Abkommens in dem Mitgliedstaat nicht bestand. So war die Rechtslage in Deutschland. ${ }^{40}$

Der Fall Soysal betrifft die aktive Dienstleistungsfreiheit, d.h. die Freiheit, eine Dienstleistung in einem anderen als dem Herkunftsstaat zu erbringen. Doch wirft die Entscheidung die
Frage auf, ob ein Visum auch zur Gewährleistung der passiven Dienstleistungsfreiheit entbehrlich ist, für den Fall also, dass türkische Staatsangehörige ins Bundesgebiet einreisen, um hier Dienstleistungen in Anspruch zu nehmen, etwa einen Sprachkurs zu besuchen und einen Sprachtest zu absolvieren. ${ }^{41}$ Das VG Berlin hat die Frage der Visumsfreiheit für die Einreise zu touristischen Zwecken (die mit der Inanspruchnahme von Dienstleistungen einhergehen) bereits grundsätzlich für möglich gehalten. ${ }^{42}$ Aller Voraussicht nach wird der EuGH, der den primärrechtlichen Grundfreiheiten ohne Ansehung des Mitgliedstaats zur weitgehenden Geltung verhilft, die Visumsfreiheit auf die passive Dienstleistungsfreiheit erstrecken. ${ }^{43}$ Dies unterstellt, setzt die Visumsfreiheit für türkische Familienangehörige allerdings immer noch voraus, dass diese erstens (nur) mit der Absicht einreisen, Kenntnisse der deutschen Sprache in entsprechenden Sprachkursen zu erwerben, und dass ihr Aufenthalt zweitens nicht von vornherein auf Dauer angelegt ist. ${ }^{44}$

Einige Aussicht auf Erfolg versprechen in einigen Bundesländern Anträge auf Erteilung einer Aufenthaltserlaubnis durch Ausländer, die mit einem Schengen-Visum bereits in die Bundesrepublik eingereist sind und nach der Einreise heiraten oder die erforderlichen Sprachkenntnisse nachweisen. Nach Auffassung des VGH Baden-Württemberg, ${ }^{45}$ dem sich andere Oberverwaltungsgerichte prinzipiell anschließen, ${ }^{46}$ kommt es für das auf $\ 39 \mathrm{Nr}$. 3 AufenthV gestützte Aufenthaltsrecht auf die ursprüngliche mit der Einreise verbundene Absicht oder die Erfüllung einiger Voraussetzungen für den Ehegattennachzug vor Einreise nicht an. Das Erschleichen eines kurzfristigen Aufenthalts unter Verschleierung der Absicht zum dauernden Aufenthalt mag hiernach zwar eine Ausweisung zu begründen, doch haben die Behörden bei der Entscheidung über eine Ausweisung die Wertentscheidung des Art. 6 GG zu beachten.

35 Ein Visum nach dem Schengener Durchführungsübereinkommen berechtigt den Inhaber während des Gültigkeitszeitraums, längstens jedoch drei Monate pro Halbjahr zum Aufenthalt im Schengenraum.

36 Anna Lübbe, ZAR 2009, 215 ff. mit Bezug auf $\$ \S 5$ Abs. 1 Nr. 3, $§ 6$ Abs. 1 Nr. 2 AufenthG i.V.m. Art. 5 Abs. 1 e), $\$ 39$ Abs. 3 Schengener Grenzkodex.

37 Siehe oben „Der Familiennachzug in Zahlen“.

38 EuGH Urt. v. 19.2.2009-C-228/o6, Vorabentscheidung

39 OVG Berlin-Brandenburg, Beschluss v. 30.3.2006, - OVG 7 B 13.05, juris.

40 Ausführlich Klaus Dienelt, ZAR 2009, 182 ff.; Hailbronner, NVwZ 2009, 760 ff.; Jan Hecker, ZAR 2009, $142 \mathrm{f}$.

41 Bejahend Vorbemerkungen einiger Abgeordneter und der Fraktion DIE LINKE in ihrer Kleinen Anfrage, BT-Drucks. 16/12562; verneinend Bundesregierung in der Antwort auf die Kleine Anfrage, BT-Drucks. $16 / 12743,3$.

42 VG Berlin, B. v. 25.2.2009 19 V 61.08, juris, m.Nw. zur Rechtsprechung des EuGH. Die Frage konnte im Ergebnis offen bleiben. Ausführlich Cornelia Mielitz, NVwZ 2009, $276 \mathrm{ff}$.

43 So z.B. auch Hailbronner, NVwZ 2009,760 (763 f.); anders die Bundesregierung, die davon ausgeht, dass der gemeinschaftsrechtliche Begriff der passiven Dienstleistungsfreiheit „,nicht direkt in den assoziationsrechtlichen Kontext übertragen werden kann“, Antwort auf eine Anfrage der Partei DIE LINKE, BD-Drucks. 16/12743, 4.

44 Hailbronner, NVwZ 2009, 760 (764 f.).

45 VGH Baden-Württemberg, B. v. 8.7.2008, $11 \mathrm{~S}$ 1041/o8, juris.

46 BayVGH B. v. 18.5.2009 - 10 CS 09.853, juris; offen gelassen von OVG Saarland, B. v. 27.2.2009 - 2 B 469/o8, juris. 
Andere Oberverwaltungsgerichte fordern allerdings weiterhin die Rückkehr der Ehepartner ins Herkunftsland und den Sprachnachweis und Visumserwerb zum Zweck des Ehegattennachzugs bei den dortigen Auslandsvertretungen. ${ }^{47}$

\section{Differenzierung der Sprachanforderungen nach Aufenthalts- status und Staatsangehörigkeit}

Verfassungsrechtlich problematisch ist auch die Differenzierung der Sprachanforderungen nach dem Aufenthaltszweck, die dazu führt, dass der zum Zweck der Beschäftigung einreisende Ausländer vor Einreise keine Sprachkenntnisse nachweisen muss, die nachziehende Ehepartnerin aber schon. ${ }^{48}$ Ähnlich problematisch ist die Differenzierung zwischen dem Nachzug zu ausländischen oder deutschen Wissenschaftlern oder zu ausländischen oder deutschen Selbständigen. ${ }^{49}$ Die Nähe zum Grundrecht von Ehe und Familie, eventuell auch zum geschlechtsspezifischen Diskriminierungsverbot, stellt strenge Anforderungen an die gebotene Gleichbehandlung, denen die Regelungen des $\mathbb{} 30$ Abs. 1 AufenthG nicht gerecht werden. Auch das Argument, gerade der Nachzug zu deutschen Ehegatten erfordere wegen des privilegierten Aufenthaltsstatus Deutschkenntnisse, vermag die genannten Differenzierungen nicht zu rechtfertigen. Wesentlich konsistenter wäre ein Konzept, das nachziehende Ehepartner ausnahmslos auf den Nachweis und gegebenenfalls Erwerb ausreichender Sprachkenntnisse nach der Einreise verpflichtet.

\section{Resümee}

Die Integrationsbedingung ausreichender Deutschkenntnisse bleibt damit problematisch. Die Integrationsbedingung ist unverhältnismäßig, insbesondere dann, wenn die Infrastruktur oder die persönlichen Verhältnisse im Herkunftsland den Spracherwerb erschweren. Sie ist außerdem unklar, was den Standard und Nachweis der sprachlichen Fähigkeiten, aber auch, was die Möglichkeiten des Erwerbs im Bundesgebiet betrifft. Der deutsche Gesetzgeber sollte auf diese Integrationsbedingung im Ehegattennachzug deshalb verzichten. Der mit dem Instrument verfolgte - ohne Zweifel wünschenswerte und förderungswürdige - Zweck des Spracherwerbs lässt sich durch das Angebot von Integrationskursen, ggf. auch durch die Verpflichtung zur Teilnahme erreichen. Der Einwand, der Spracherwerb sei nach Zuzug ins Bundesgebiet nur noch schwer durchzusetzen, ist wenig stichhaltig. Das Beispiel des Aufnahme- und Integrationsvertrags in Frankreich zeigt, dass Instrumente zur Verfügung stehen, mit denen sich die gesetzlichen Ziele auf andere, für die Ehepaare mildere Weise durchsetzen lassen.

47 Vgl. HessVGH, B. v. 22.9.2008 - 1 B 1628/o8, juris; OVG RheinlandPfalz, B. v. 20.4.2009-7 B 10037/og, juris; OVG Lüneburg, B. v. 27.7.2009-11 ME 171/og, juris; anders noch OVG Lüneburg, B.v. 28.8.2008-11 ME 131/08, juris.

48 Kingreen, ZAR 2007, 13 (19).

49 Ausführlich Henning J. Thomas, SächsVBI. 2009, 56 (59 f.).

\section{Probleme der Vereinbarkeit von Scharia-geprägtem Familienrecht mit deutschem Recht}

Beatrix Weber-Monecke,

Richterin am Bundesgerichtshof, Karlsruhe

Zunächst möchte ich einen kurzen Streifzug durch islamisches Familienrecht, insbesondere durch das Scheidungsrecht, unternehmen. Im Islam ist die Scheidung (arab.: talaq) das Auflösen eines Rechtsverhältnisses, das durch den privatrechtlichen Vertrag der Eheschließung zustande gekommen ist. ${ }^{1}$ Die Scheidung ist eine einseitige Rechtshandlung, die vom Ehemann oder einem Stellvertreter vorgenommen werden kann und die zu ihrer Gültigkeit in Anwesenheit von zwei Zeugen erfolgen muss. Eine Ehefrau kann die Scheidung nur begehren, wenn ihr Ehemann ihre zwingenden Rechte verletzt hat. ${ }^{2}$

\section{Überblick über Scharia-geprägtes Scheidungsrecht am Bei- spiel des islamisch-iranischen Rechts \\ Das iranische Recht, mit dem ich mich exemplarisch befassen will, sieht ausschließlich die gerichtliche Ehescheidung vor. Es gibt sogenannte zivile Sondergerichte, die für Personenstands- sachen, daher insbesondere auch für Scheidungen, zuständig}

sind. Dem Gericht sitzen männliche Richter vor; daneben können weibliche Beraterinnen berufen werden, was in der Praxis dem Vernehmen nach auch geschieht. Dem Antrag auf Scheidung folgt ein Verfahren, in dem Schiedsrichter ernannt werden, die die Parteien zu beraten und auf die Beilegung der Streitigkeiten hinzuwirken haben. Dieses Verfahren ist unabhängig davon einzuhalten, ob ein Mann oder eine Frau die Scheidung begehrt. ${ }^{3}$ Im iranischen ZGB ist die Scheidung für Muslime in Anlehnung an das islamisch-schiitische Recht als Mannesrecht geregelt. Ein Mann kann ohne Vorliegen und Angabe von Gründen seine Frau verstoßen. ${ }^{4}$ Das Scheidungsbegehren einer Frau ist dagegen davon abhängig, dass Scheidungsgründe vorliegen. Deshalb spielt das materielle Scheidungsrecht insbesondere in dieser Hinsicht eine Rolle.

1 Zum Eheschließungsvertrag im iranischen Recht: Bergmann/Ferid/ Henrich/Enayat, Internationales Ehe- und Kindschaftsrecht, Länderteil Iran, Ergänzungslieferung 158, $37 \mathrm{f}$.

2 Yassari, FamRZ 2002, 1088; Elwan/Menhofer STAZ 2005, 168, 172 f.

3 Vgl. Yassari, FamRZ 2002, 1088, 1089 f.; Bergmann/Ferid/Henrich/ Enayat, a.a.O., $63 \mathrm{ff}$.

4 Art. 1133 iran. ZGB. 